\title{
DIFFERENTIAL EXPRESSION ANALYSIS OF KRUPPEL LIKE FACTORS 6 AND ANTAGONISTIC EFFECT STUDY OF CINNAMIC ACID - AN IN SILICO APPROACH
}

JEMMY CHRISTY H*, SWETHA V

Department of Bioinformatics, Sathyabama University, Chennai, Tamil Nadu, India. Email: jemmychristy.bioinfo@sathyabama.ac.in

Received: 07 April 2019, Revised and Accepted: 13 May 2019

\section{ABSTRACT}

Objective: Krüppel-like factor 6 (KLF6) is the significant member of a DNA binding proteins which mainly involved in the transcriptional regulation as well various promising cellular processes such as cell proliferation and differentiation, cytokine signal-based inflammatory responses, and pluripotency activity of cells. Our computational studies involve KLF6 differential expression in breast cancer tissues based on web resources such as Oncomine and cBioportal.

Methods: Oncomine and TCGA data-based CBioportal were the databases used to explore the KLF6 expression, and KLF6 was underexpressed in many of the breast cancer tissues than normal breast tissues. Major breast cancer datasets such as Curtis and TCGA supported the clinical-pathological role of KLF6, mutational frequencies. Further prognosis analysis was carried out using Survexpress and it revealed the survival rate and risk group categorization. Thus, KLF6 was considered as a therapeutic target and natural compound cinnamic acid antagonistic efficacy was analyzed based on molecular docking and simulation studies.

Results: Systematic analysis of KLF6 gene expression in breast cancer would be helpful in exploring aspects of KLF6 as the potential drug target as well as prognostic disease marker identification. Molecular docking and dynamic study were carried out to evaluate the intermolecular interaction between the cinnamic acid and KLF6 and the docked complex stability after 5ns.

Conclusion: Thus, the computational study demonstrated the cinnamic acid role as an anticancer compound to combat the overexpression of KLF6 to combat cancer. Further, in vitro and in vivo studies need to be carried out to know the insights of antagonistic effect.

Keywords: Krupple-like factor, Breast cancer, Gene expression, Survival analysis, Cinnamic acid, Anticancer.

(c) 2019 The Authors. Published by Innovare Academic Sciences Pvt Ltd. This is an open access article under the CC BY license (http://creativecommons. org/licenses/by/4. 0/) DOI: http://dx.doi.org/10.22159/ajpcr.2019.v12i7.33406

\section{INTRODUCTION}

Worldwide cancer statistics explored the breast cancer prevalence among the woman and corresponding physical and mental trauma. Breast cancer is one of the top listed tumor threats and their incidence is observed in males also. Prognosis level of few breast cancer subtypes showed low survival rate [1]. Krüppel-like factors (KLFs) play a key role as transcriptional regulators and play a crucial role in the cell cycle system such as proliferation, differentiation, inflammatory responses, migration of cells, and pluripotent ability $[2,3]$. KLF6 related somatic mutations frequency is prevalent in prostate cancer, due to that tumor-suppressing ability become inactive [4,5]. Various clinical studies on major cancers such as colorectal [6-13] specialized lung cancer [9-14] gastric cancer [10-15], head and neck cancer subtypes[11-16], hepatocarcinoma, and ovarian cancer. Thus, KLF6 relationships were investigated and revealed the gene differential expression. Based on tissue and cell types KLF6s native growth suppression properties reported in the major cancer pathways such as p53-independent upregulation of p21, disruption of cyclin D1 [12-17], and CDK4 interaction, induction or protection of apoptosis, and c-Jun inhibition [13]. Clinical evidence showed the two-way correlation between ER- $\alpha$ and KLF6 expression levels in ERpositive breast cancer. KLF6 plays a crucial role in cell growth, cell differentiation, cell adhesion, and endothelial motility depending on cell type and context. Clinical studies proposed the hypotheses that KLF6 are the significant regulator in estrogenic receptor-mediated deregulation in breast cancer tissues. Functional enrichment and deregulation gene expression pattern studies of KLF6 would be helpful to explore the new insights in therapeutics as well as diagnosis field. Identifying the potential disease marker that plays a critical role in metastasis and tumorigenesis using systemic computational analysis would be helpful in exploring the treatment regimen in case of cancer treatment. In addition to, the functional enrichment studies of KLF6 among breast cancer patients, our studies include studies on cinnamic acid antagonistic efficacy on KLF6. Natural compounds considered as anticancer agents in inducing apoptotic effect as well as a cytotoxic effect on cancer cells [14]. Cinnamic acid is one of the plant-derived compound and belongs to the auxin family and mainly involved in cell growth, differentiation, and regulations [15]. Cinnamates natural hydroxyl compounds were known for their antitumor activity and they consist of aromatic fatty acid compiled with phenyl ring substituted with the acrylic group and exist as a trans compound with low toxicity [16,17]. Cinnamic acid also contains an $\alpha, \beta$ unsaturated carbonyl group that act s Michael receptor, i.e. an active moiety considered a promising feature for anticancer drugs [18]. Recent studies on cinnamic acid usage as medicinal compound observed that cinnamic acid as well as its analog's caffeic acid, sinapic acid, ferulic acid, and isoferulic acid were exhibiting immune modulatory, antiinflammatory, and anticancer properties [19].

\section{MATERIALS AND METHODS}

\section{Data mining of KLF6 differential expression pattern}

Assessment of differential gene expression pattern of KLF6 gene in breast cancer was carried out based on various datasets compiled in the Oncomine [20] database (https://www.oncomine.org). This specialized web-based data mining tool consists of genome-wide microarray data from published as well as publically available data resources. As of October 2017, Oncomine composed of 715 datasets and 86,733 samples; the database also has 12,764 normal samples. Datasets included the mRNA expression or DNA copy number from various tumors, cell lines, and published research articles. Our query gene information's were 
retrieved from 191 datasets and 33,630 samples (Oncomine). Clinical data with statistical evidence would be helpful in exploring the cancerrelated genes, differential expression and prognosis analysis. Analysis of KLF6 gene differential expression levels in various cancer tissues initiated using keyword search and filter module options. All data in the selected datasets were log-transformed, median centered per array, and standard deviation normalized to one per array. Differentially expressed genes were retrieved from the datasets, namely based on $\mathrm{p}$ value and fold change [21,22].
Differential expression frequency of KLF6 in various breast cancer subtypes

An online portal for cancer genomic data, namely cBioportal, was used to retrieve the integrated gene expression data of KLF6 and their role in ontogenesis as well as their clinical outcomes. This updated web portal contains recent TCGA published datasets along with provisional TCGA datasets [23,24]. Based on the multiple datasets compiled in the cBioportal KLF6 role in breast cancer explored further and mutational patterns among the breast cancer tissues were assessed.
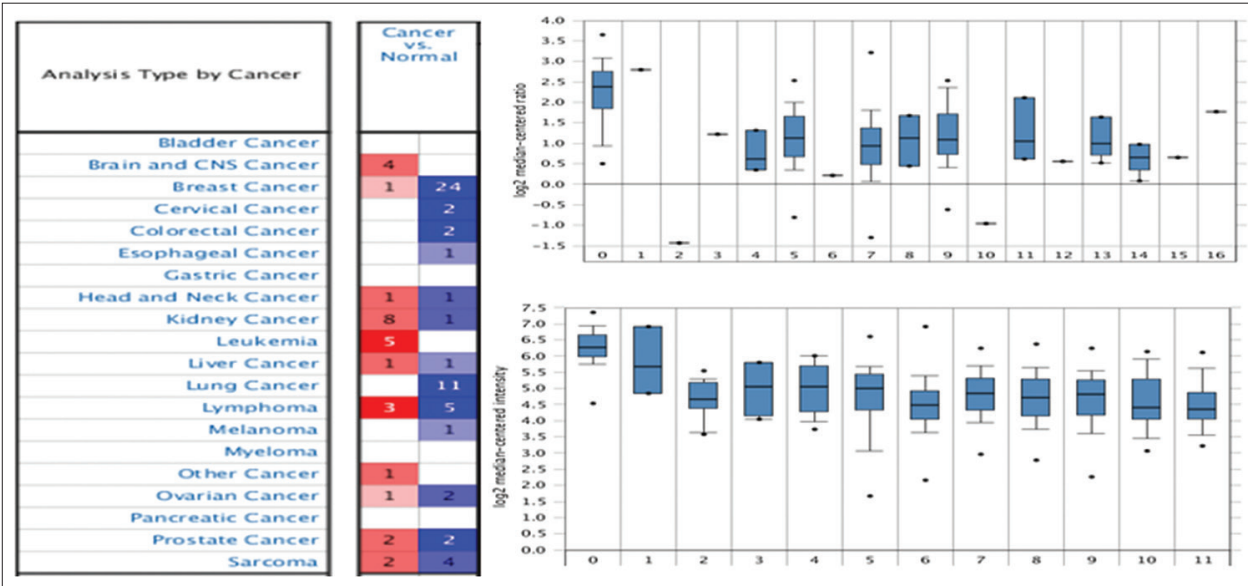

Fig. 1: The mRNA expression patterns of Krüppel-like factor 6 in cancers

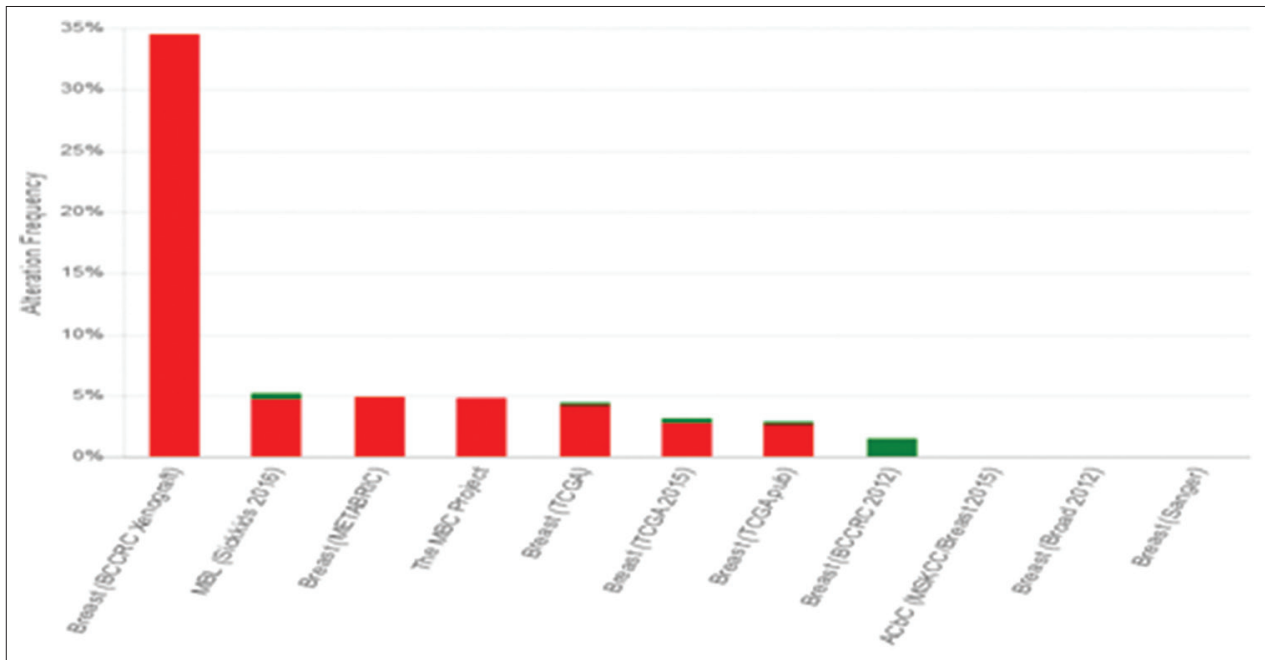

Fig 2: Alteration frequency of KLF6 among breast cancer datasets and breast cancer types

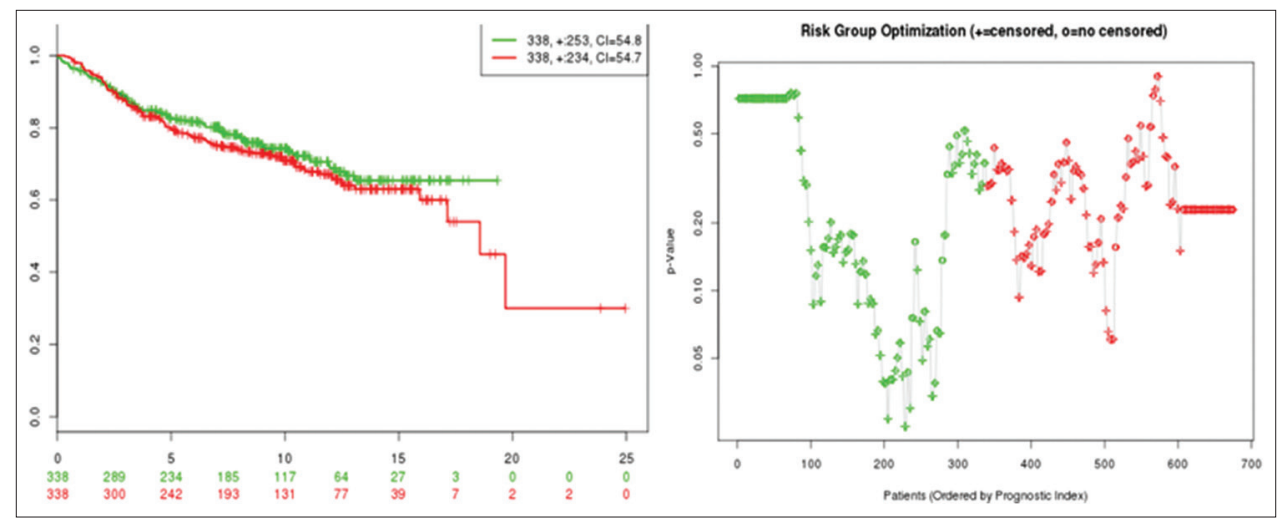

Fig 3: KM-Plot based prognosis analysis for KLF6 in breast cancer samples 
Table 1: Mutational changes reported for Krüppel-like factor 6

\begin{tabular}{lll}
\hline Sample ID & Cancer type & Protein change \\
\hline TCGA-C8-A1HM-01 & Breast invasive ductal carcinoma & K80N \\
TCGA-JL-A3YW-01 & Breast mixed ductal and lobular carcinoma & D107Cfs*7 \\
SA092 & Invasive breast carcinoma & S142A \\
MBC_49 & Invasive breast carcinoma & T179P \\
TCGA-C8-A1HM-01 & Breast invasive ductal carcinoma & K80N \\
TCGA-JL-A3YW-01 & Breast mixed ductal and lobular carcinoma & D107Cfs*7 \\
TCGA-C8-A1HM-01 & Breast invasive ductal carcinoma & K80N \\
\hline
\end{tabular}

Table 2: Functional enrichment analysis based on pathways

\begin{tabular}{ll}
\hline Pathway & $\mathbf{p}$ \\
\hline DNA binding & 0.000369 \\
Transcription from RNA polymerase ii promoter & $6.59 \mathrm{E}-05$ \\
Positive regulation of transcription, DNA-dependent & $7.28 \mathrm{E}-05$ \\
Transcription factor binding & $9.55 \mathrm{E}-06$ \\
Negative regulation of transcription, DNA-dependent & 0.000623 \\
Protein domain specific binding & 0.0016 \\
Protein binding transcription factor activity & 0.00217 \\
Identical protein binding & 0.0126 \\
Protein dimerization activity & 0.0181 \\
Enzyme binding & 0.037 \\
\hline
\end{tabular}

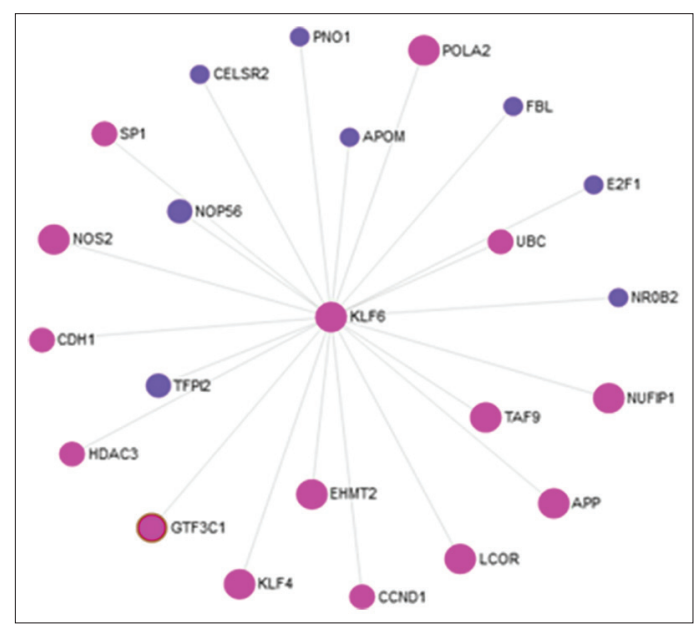

Fig. 4: KLF6 and associated genes based on pathway enrichment analysis

Network construction for KLF6 based on pathway enrichment analysis

To identify the molecular signature as well their biological processes of KLF6 and associated genes, we used network analyst tool [25], a meta-analysis tool meant for assessing the gene expression data based statistical parameters. It enables the researchers to find the significant features, connections, patterns, and new functional hypotheses. Proteinprotein interaction networks are the undirected graphs embedded with nodes (Gene/Protein) and edges (Interaction). Our computational studies considered the betweenness centrality of a node among the KLF6 associated genes.

\section{Prognosis analysis of KLF6}

Computational tool Surv express was used to analyse the prognosis level and associated risk of KLF6 expression in breast cancer patients [26].

\section{Homology modeled KLF6 structure and structure validation} KLF6 three-dimensional structure was modeled in MODELLER. Its a comparative modeling tool can automatically calculate a model containing all non-hydrogen atoms in a faster manner. Not only model building, in addition to that MODELLER, but can also perform alignment of two or more protein sequences and/or structures or their fold-assignment [27], clustering of sequences and or structures and $\mathrm{Ab}$ Initio modeling of loops in protein structures [28]. The model is then optimized depending on conjugate gradients method, and molecular dynamics simulation is performed to minimize errors of the spatial restraints.

\section{Targeting the KLF6 with cinnamic acid}

Literature survey on cinnamic acid and its natural analogs revealed that they possess unique anticancer properties; thus, they can act as a potent anti-proliferative agent. In our studies, we target the KLF6 with cinnamic acid to evaluate the intermolecular interaction based subsequent antagonist effect. Molecular docking approach was used to evaluate the intermolecular interactions between cinnamic acid and KLF6. Molecular docking involves ligand preparation and physiochemical properties and dug likeliness evaluation. Cinnamic acid structure three-dimensional structure in SDF format was retrieved from PubChem, a publically available resource for chemical structures [29]. A potent molecular which could act like a drug on an appropriate target should be in sufficient concentration and as well as in bioactive form to carry out specified action. In addition to this absorption, distribution, metabolism, and excretion and toxicity (ADMET) properties were assessed for cinnamic acid using Swiss ADME and Discovery studio ADMET screening. Swiss ADME, a web browser was used to for the ADME screening, along with physiochemistry, drug-likeness, pharmacokinetics, and medicinal chemistry friendliness properties assessment [30].

Molecular docking is a method for analyzing the preferred orientation within the active site of KLF6 to an as well as low energy binding mode of cinnamic acid when bound to each other to form a stable complex. Preparation such as forcefield assignment (CHARMM) and binding site prediction (Eraser algorithm) was done to initiate molecular docking; docking was performed using ligand fit algorithm of Discovery studio. Ligand fit performs the docking in two stages, namely specifying the region within the KLF6 receptor to define an active site and then docking the cinnamic acid on the specified site [31].

\section{Intermolecular assessment of cinnamic acid using ligand fit}

Docked KLF6 and cinnamic acid complexes were subjected for in situ ligand minimization to minimize the ligand energy level with the receptor cavity of KLF6 docked complex, then scoring functions used to evaluate the favorable binding interaction based on their energy; negative or low energy level of docked complexes confined with stable conformation. Six distinct scoring functions, namely LigScore1, LigScore2,-PLP1, PLP2, Jain, and PMF were employed to evaluate the docking poses score and consolidated dock score were analyzed to rank the best-docked complex [32]. The scoring functions, PLP1, PLP2, and PMF, were evaluated by the sum of the two types of the pairwise interaction, namely $\mathrm{H}$ bond and steric interactions, between the protein and the compound [33-35].

Molecular dynamics and simulation of KLF6 and cinnamic acid docked complexes

Molecular dynamics and simulation efficiently explore the stability of the docked complex, since stable interaction between the ligand and receptor molecule could render the specified antagonistic activity on the target. Here, we explored the KLF6 and cinnamic acid structural stability in the implicit solvent environment. 


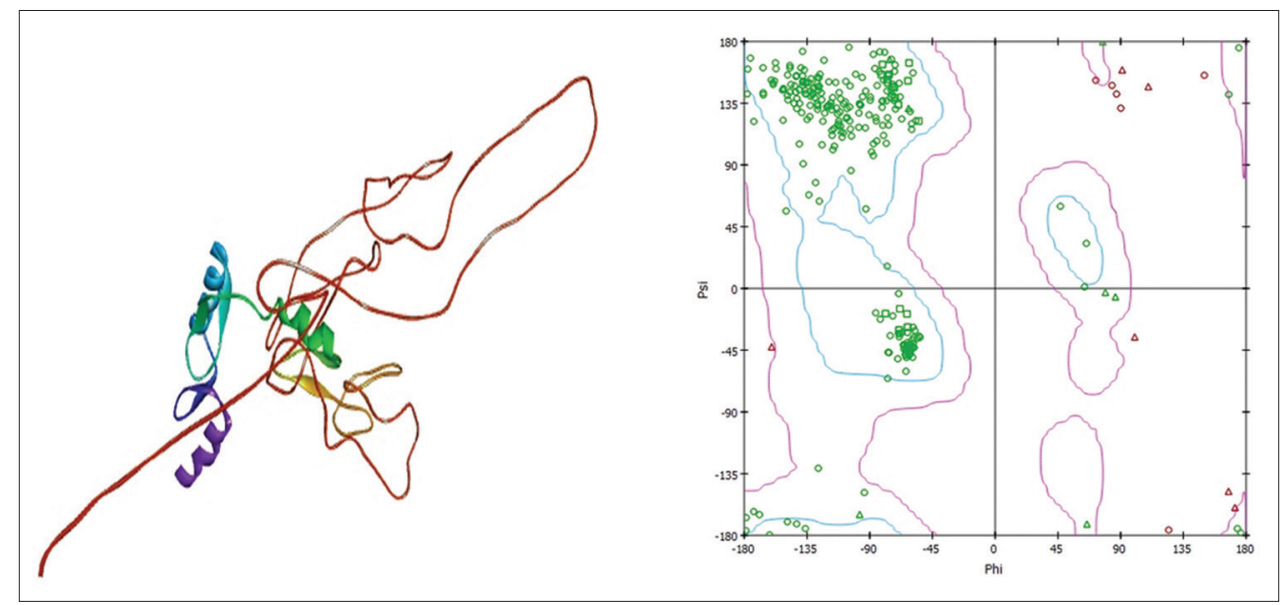

Fig. 5: Modeled KLF6 structure and quality evaluation based on Ramachandran plot

Table 3: Intermolecular interaction of cinnamic acid and Krüppel-like factor 6

\begin{tabular}{lll}
\hline $\begin{array}{l}\text { Receptor-ligand } \\
\text { interaction }\end{array}$ & Distance & $\begin{array}{l}\text { Intermolecular } \\
\text { interaction bond type }\end{array}$ \\
\hline $\begin{array}{l}\text { Cinnamic Acid: } \\
\text { H19 - GLU235: O }\end{array}$ & 3.03612 & Hydrogen bond \\
$\begin{array}{l}\text { SER270: HN - Cinnamic } \\
\text { acid: O2 }\end{array}$ & 2.27768 & Hydrogen bond \\
$\begin{array}{l}\text { ARG271: HN - Cinnamic } \\
\text { acid: O2 }\end{array}$ & 2.36241 & Hydrogen bond \\
$\begin{array}{l}\text { HIS274: HD1 - Cinnamic } \\
\text { acid: O2 }\end{array}$ & 2.06956 & Hydrogen bond \\
$\begin{array}{l}\text { SER270: HB1 - Cinnamic } \\
\text { acid: O2 }\end{array}$ & 2.28391 & Hydrogen bond \\
$\begin{array}{l}\text { GLU235: OE2 - Cinnamic } \\
\text { acid }\end{array}$ & 3.01115 & Electrostatic \\
$\begin{array}{l}\text { Cinnamic acid - PHE269 } \\
\text { Cinnamic acid - CYS268 }\end{array}$ & 5.7312 & Hydrophobic \\
\hline
\end{tabular}

Molecular dynamics and simulation studies carried out using standard dynamics cascade module of discovery studio at v2.1. Modeled KLF 6 structure was applied with CHARMM forcefield [36] to assign the atom and residue position and also to evaluate the missing atoms using Momany Rone potential charge estimation method. Before production step equilibration step carried out to allow the complex to evolve spontaneously for a period of time as well as integrating the equations of motion until the average temperature and structure remained stable, and the total energy converged particle Mesh Ewald algorithm was used to assess the long-range electrostatic interactions. Shake algorithm was used to analyze the hydrogen atoms constraints. Docked KLF6 and cinnamic acid complex were solvated within a preequilibrated orthorhombic box of TIP3P water along with $0.145 \mathrm{~mol} / \mathrm{L}$ $\mathrm{NaCl}$ concentration. The docked complex was applied with periodic boundary conditions, and the system was minimized for 2000 steps based on conjugant gradient method as well as steepest descent method. Minimized complex was subjected for standard dynamics cascade steps, namely heating, equilibration, and production in sequence. Simulation carried out for five ns and temperature of the simulation system was up to 300k and keeping the KLF6 and cinnamic acid complex with a harmonic force constant of $0 \mathrm{kcal} / \mathrm{mol} / \AA 2$. Further equilibration step was performed for 400 PS with a constant pressure of $1 \mathrm{~atm}$. Finally, without any constraints, the production step was performed for five ns at a constant temperature of $300 \mathrm{~K}$ and a constant pressure of $1 \mathrm{~atm}$ [37]. The obtained trajectory snapshots of KLF6 and cinnamic acid complex was saved every 2 ps for further analysis.

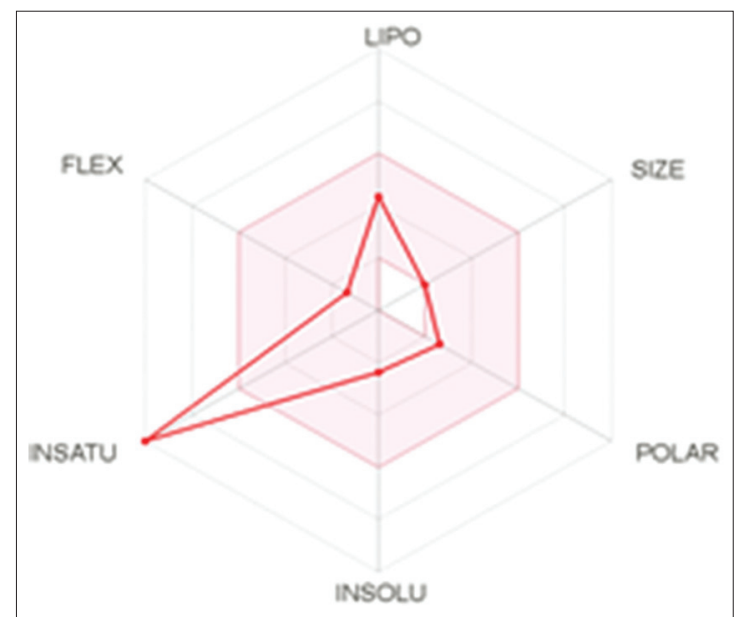

Fig. 6: Physicochemical, pharmacokinetic, drug-like properties of cinnamic, acid and its derivatives

\section{RESULTS AND DISCUSSION}

Oncomine data portal was used to explore the KLF6 gene expression pattern; nearly 191 datasets that are composed of 33,630 samples were assessed in our computational analysis. Based on the statistical parameters such as $p<0.05$ and fold change of $>2$, the differentially expressed gene patterns were screened. Retrieved data were highlighted in the following figure, each number in the colored cells represent the number of breast cancer datasets that are within the specified thresholds. Overexpressed breast cancer datasets with KLF6 were highlighted with red color and blue color were meant for underexpressed breast cancer datasets with KLF6. Based on gene rank color intensities varies, thus darker color specifies the better percentile (Fig. 1).

Comprehensive Oncomine analysis of breast cancer dataset revealed the varied gene expression levels of KLF6 among breast cancer tissues such as overexpression and underexpression, but clinic pathological datasets supported the underexpression pattern association with breast cancer than overexpression frequency. Two major datasets, namely Curtis breast dataset (2136 samples and 19,273 measured genes) and TCGA breast dataset (593 samples and 20,423 measured genes) supported the underexpression of KLF6 among the various breast cancer subtypes, especially lobular and ductal types Fig. 1. Gene expression variation represented in box plots that compare the KLF6 expression in normal (left plot $/ 1^{\text {st }}$ plot) and cancer tissues.

Comprehensive Oncomine analysis of breast cancer dataset revealed the varied gene expression levels of KLF6 among breast cancer tissues, but 


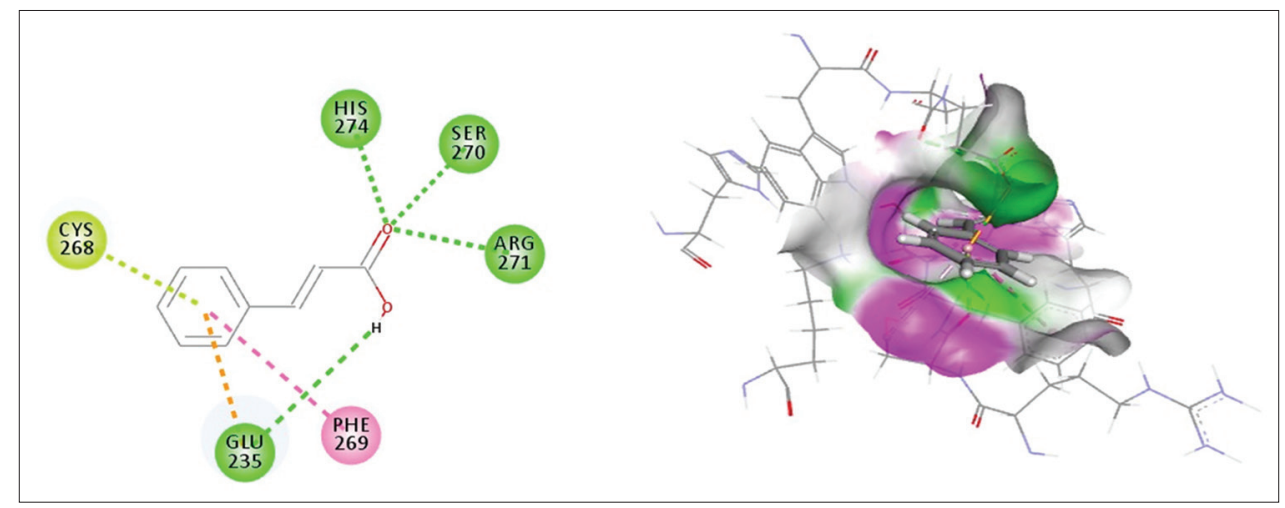

Fig. 7: Intermolecular interaction of cinnamic acid on KLF6

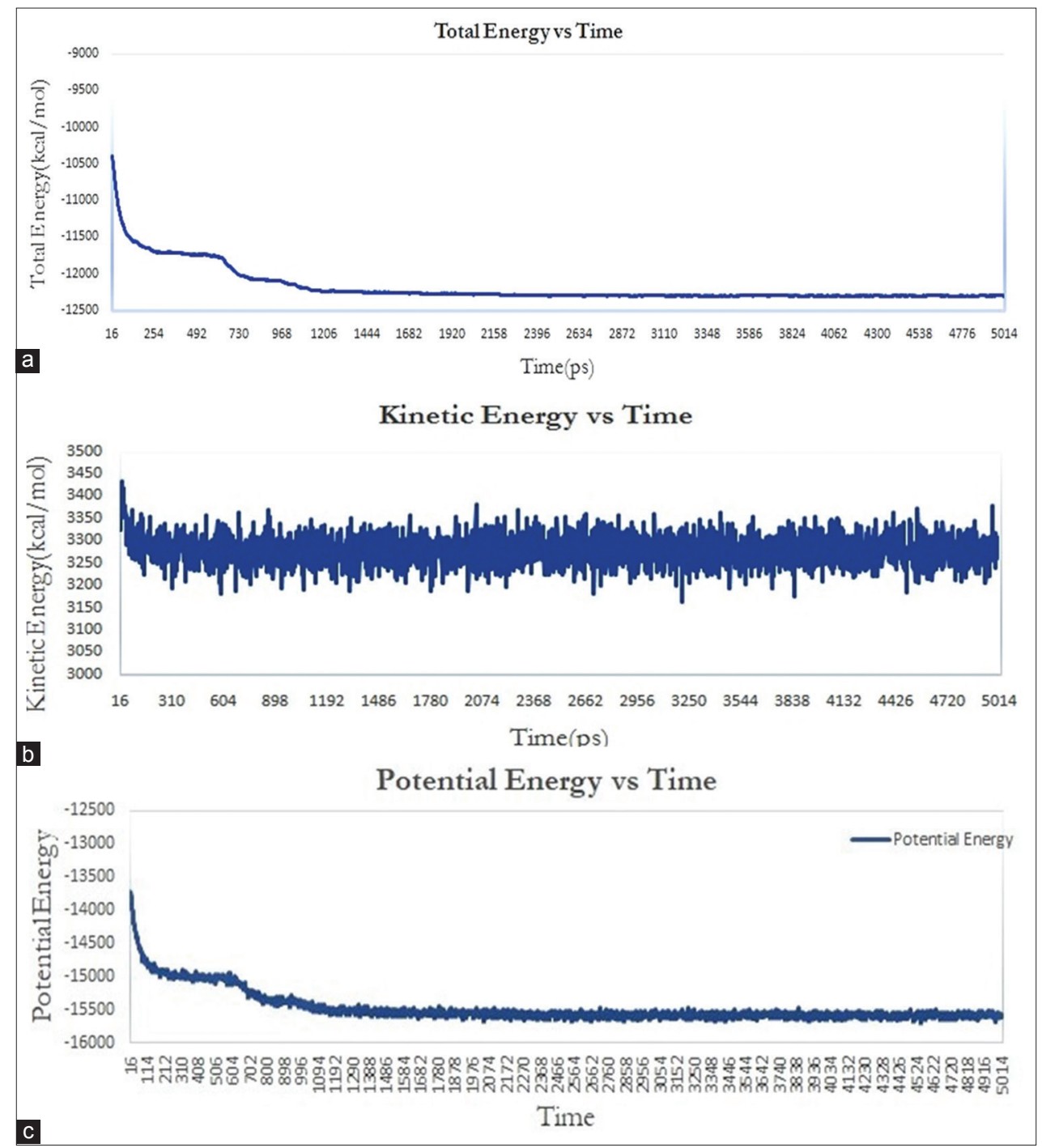

Fig. 8: (a-c) Total, kinetic, and potential energy fluctuations of KLF6 and cinnamic acid docked complexes

clinical datasets supported the under-expression pattern association with breast cancer than over expression frequency. Two major datasets namely Curtis breast dataset (2,136 samples and 19,273 measured genes) and TCGA breast dataset (593 samples and 20,423 measured genes) supported the under expression of KLF6 among the various breast cancer subtypes especially lobular and ductal types Fig. 1. Gene expression variation represented in box plots that compares the KLF6 expression in normal (left plot $/ 1^{\text {st }}$ plot) and cancer tissues.
Differential expression frequency of KLF6 in various breast cancer subtypes

Alteration frequencies of KL6 among 11 recent datasets, namely Adenoid Cystic Carcinoma of the Breast (MSKCC, J Pathol. 2015), Breast Cancer (METABRIC, Nature 2012 and Nat Commun 2016), Breast Invasive Carcinoma (British Columbia, Nature 2012), Breast Invasive Carcinoma (Broad, Nature 2012), Breast Invasive Carcinoma (Sanger, Nature 2012), Breast Invasive Carcinoma (TCGA, Cell 2015), Breast Invasive Carcinoma 


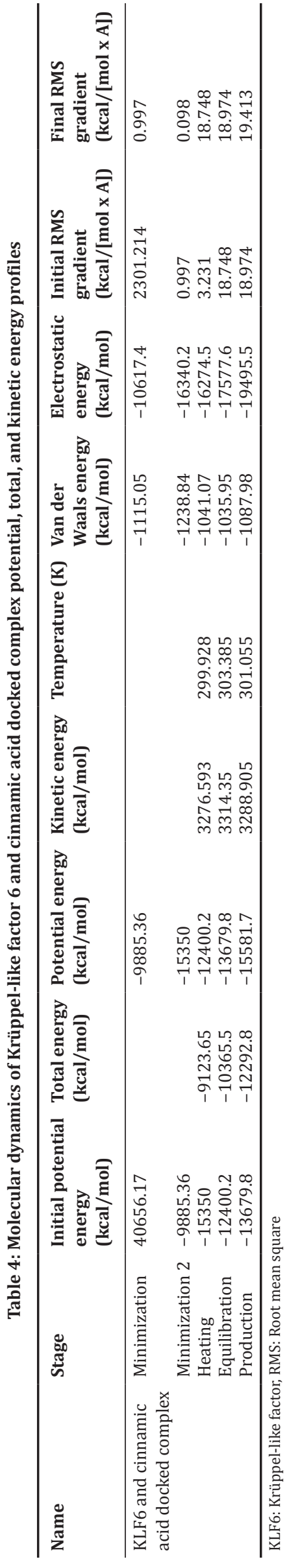

(TCGA, Nature 2012), Breast Invasive Carcinoma (TCGA, Provisional), Breast cancer patient xenografts (British Columbia, Nature 2014), Mutational profiles of metastatic breast cancer (France, 2016), and The Metastatic Breast Cancer Project (Provisional, October 2017) were highlighted in Fig. 2. Based on the 11 distinct datasets analysis, it was observed that KLF6 varied response is more prevalent in invasive ductal carcinoma than invasive lobular carcinoma. KLF6 mutational frequencies revealed that four missense and two frameshift changes (Table 1)

\section{Prognosis analysis}

$\mathrm{Km}$-plot revealed the $\mathrm{CI}$-concordance Index and $\mathrm{p}$ value used in the analysis. CI determined the probability of the subjects with high risk, red and green line was meant for high- and low-risk groups associated with breast cancer. In general, the red and green numbers next to the horizontal axis represent the number of number of individuals not mentioned in the risk group a long time. The risk plots help the user to visualize how the risk groups partitions were made on SurvExpress to generate the Kaplan-Meier plots (Fig. 3). Categorization of risk group partitions was made to generate KM-Plot, its a simple way to assess the variations in the $p$ value if any deviations were observed in the analysis. But by default, this prognosis analysis tool split the risk group by the median of the prognostic index generating risk groups of a similar number of samples.

Network construction and enrichment analysis based on molecular function

KLF6 and associated genes network was constructed using network analyst, based on degrees and betweenness centrality top listed interacting partners for KLF6 were included in the enrichment studies. KLF6 and related genes involved in the diverged function DNA binding to transcription initiation to protein dimerization activity. All these specified functions were assessed based on p value cutoff and highlighted with different color (Fig. 4). Underexpression of these crucial genes generate the deregulated pathways and end up in differential gene expression. DNA binding, transcription from RNA polymerase ii promoter, positive regulation of transcription, DNA-dependent, transcription factor binding, negative regulation of transcription, DNA-dependent, negative regulation of transcription, DNA-dependent, protein domain specific binding, protein binding transcription factor activity, identical protein binding, protein dimerization activity, and enzyme binding were the major GO terms based functions KLF6 (Table 2).

\section{KLF6 structural insights}

KLF6 structure was modeled using the homology modeling approach to evaluate the therapeutic potential of cinnamic acid. Modeler tool based comparative modeled structure was analyzed for stereochemical properties using rampage tool, and mol probity tool was used to assess the steric hindrance related clash score calculations. The model evaluation suggested that the preferred model and KL6 model were subjected for binding site analysis, there were totally nine binding sites and larger binding site was chosen for cinnamic acid interaction (Fig. 5).

\section{Drug likeliness and ADME screening of cinnamic acid}

Computational methods are being used to analyze the cinnamic acid based on different molecular characteristics to assess its drug-likeness. Pharmacokinetic and ADMET (absorption, distribution, metabolism, excretion, and toxicity) screening were done using Swiss ADME tool (Fig. 6). Swiss ADME assessment involves six physicochemical properties, namely lipophilicity, size, polarity, solubility, flexibility, and saturation. Radar plot with pink area depicted the physiochemical range of cinnamic acid and their drug likeliness. Except for moderate saturation properties, others were within the optimal range. The molecular properties weight $<500 \mathrm{~g} / \mathrm{mol}$, minimum of five hydrogen bond donors and ten hydrogen bond acceptors whereas $<10$ rotatable bonds were chosen as criteria for assessing the cinnamic acid therapeutic properties.

Molecular docking and dynamics simulation studies of cinnamic acid on KLF6

The receptor ( $\alpha$ - $\delta$-Bgt- 4 toxin) and selected ligand molecules were docked using the Ligand-Fit algorithm of Discovery Studio 2.0. Receptor KLF6 and cinnamic acid molecule was docked using Ligand-Fit algorithm in 
the discovery studio 2.0 platform.Intermolecular interaction profile of interacting residues and bonding distance were listed in Table 3 and Fig. 7. Dock score calculated based various scoring functions and interactions observed with higher dock score. Further docked complexes was subjected to a MD simulation for a period of $5 \mathrm{~ns}$.Energy profiles of docked complex were listed in the Table 4 and Fig. 8 Initial and final potential energy ,kinetic and total energy profile were listed as graph and it was observed that stable energy level was achieved around $-12230 \mathrm{kcal} / \mathrm{mol}$. From Simulation studies it is evident that hydrogen bond interaction were stable after $3 \mathrm{~ns}$.

\section{CONCLUSION}

Our findings showed that the differential patterns and frequency of the KLF6 mutations in breast cancer are similar to the mutation profiles which are observed in other cancer types. However, these mutations do not seem to affect the expression level of the gene which is frequently downregulated in tumor tissues. Downregulation of the KLF6 mRNA expression may be modulated by the effect of epigenetic regulations. The systematic study revealed the centrality of the KLF6 in the specified pathway, so considering KLF6 as a drug target can pave the dynamic role in cancer biology. In addition to this differential expression studies, cinnamic acid antagonist ability was tested on KLF6. Intermolecular interaction assessment was carried out by molecular dynamics, and simulation studies revealed the promising interaction mediated by conventional hydrogen bonding as well as electrostatic bonding. The information represented in this systemic study revealed that cinnamic acid and its derivatives can be used as a potent modulator for KLF6 expression in different kinds of cancer tissues.

\section{AUTHORS' CONTRIBUTIONS}

Jemmy Christy and Swetha are equally contributed the manuscript content.

\section{CONFLICTS OF INTEREST}

Authors declare that they have no conflicts of interest.

\section{REFERENCES}

1. Goldhirsch A, Winer EP, Coates AS, Gelber RD, Piccart-Gebhart M, Thürlimann B, et al. Personalizing the treatment of women with early breast cancer: Highlights of the St Gallen international expert consensus on the primary therapy of early breast cancer 2013. Ann Oncol 2013;24:2206-23.

2. Kaczynski J, Cook T, Urrutia R. Sp1-and krüppel-like transcription factors. Genome Biol 2003;4:206.

3. Yamanaka S. Strategies and new developments in the generation of patient-specific pluripotent stem cells. Cell Stem Cell 2007:1:39-49.

4. Yang Y, Nakagawa H, Tetreault MP, Billig J, Victor N, Goyal A, et al. Loss of transcription factor KLF5 in the context of p53 ablation drives invasive progression of human squamous cell cancer. Cancer Res 2011;71:6475-84.

5. Narla G, Heath KE, Reeves HL, Li D, Giono LE, Kimmelman AC, et al. KLF6, a candidate tumor suppressor gene mutated in prostate cancer. Science 2001;294:2563-6.

6. DiFeo A, Narla G, Hirshfeld J, Camacho-Vanegas O, Narla J, Rose SL, et al. Roles of KLF6 and KLF6-SV1 in ovarian cancer progression and intraperitoneal dissemination. Clin Cancer Res 2006;12:3730-9.

7. Teixeira MS, Camacho-Vanegas O, Fernandez Y, Narla G, DiFeo A, Lee B, et al. KLF6 allelic loss is associated with tumor recurrence and markedly decreased survival in head and neck squamous cell carcinoma. Int J Cancer 2007;121:1976-83.

8. Benzeno S, Narla G, Allina J, Cheng GZ, Reeves HL, Banck MS, et al. Cyclin-dependent kinase inhibition by the KLF6 tumor suppressor protein through interaction with cyclin D1. Cancer Res 2004;64:3885-91.

9. Miyaki M, Yamaguchi T, Iijima T, Funata N, Mori T. Difference in the role of loss of heterozygosity at 10p15 (KLF6 locus) in colorectal carcinogenesis between sporadic and familial adenomatous polyposis and hereditary nonpolyposis colorectal cancer patients. Oncology 2006;71:131-5.

10. Sirach E, Bureau C, Péron JM, Pradayrol L, Vinel JP, Buscail L, et al. KLF6 transcription factor protects hepatocellular carcinoma-derived cells from apoptosis. Cell Death Differ 2007;14:1202-10.

11. Slavin DA, Koritschoner NP, Prieto CC, López-Díaz FJ, Chatton B, Bocco JL, et al. A new role for the kruppel-like transcription factor
KLF6 as an inhibitor of c-jun proto-oncoprotein function. Oncogene 2004;23:8196-205.

12. Liu J, Du T, Yuan Y, He Y, Tan Z, Liu Z, et al. KLF6 inhibits estrogen receptor-mediated cell growth in breast cancer via a c-src-mediated pathway. Mol Cell Biochem 2010;335:29-35.

13. Gehrau RC, D'Astolfo DS, Dumur CI, Bocco JL, Koritschoner NP. Nuclear expression of KLF6 tumor suppressor factor is highly associated with overexpression of ERBB2 oncoprotein in ductal breast carcinomas. PLoS One 2010;5:e8929.

14. Paul S, Kundu R. Induction of apoptosis by fatty acid rich fraction of Solanum nigrum on cervical cancer cell lines. Int J Pharm Pharm Sci 2017:9:199-206.

15. Hoskins JA. The occurrence, metabolism and toxicity of cinnamic acid and related compounds. J Appl Toxicol 1984;4:283-92.

16. Kroon PA, Williamson G. Hydroxycinnamates in plants and food. J Sci Food Agric 1999;79:355-61.

17. Ahn BZ, Sok DE. Michael acceptors as a tool for anticancer drug design. Curr Pharm Design 1996;2:247-62.

18. Zou HB, Dong SY, Zhou CX, Hu LH, Wu YH, Li HB, et al. Design, synthesis, and SAR analysis of cytotoxic sinapyl alcohol derivatives. Bioorg Med Chem 2006;14:2060-71.

19. Su P, Shi Y, Wang J, Shen X, Zhang J. Anticancer agents derived from natural cinnamic acids. Anticancer Agents Med Chem 2015;15:980-7.

20. Rhodes DR, Yu J, Shanker K, Deshpande N, Varambally R, Ghosh D, et al. ONCOMINE: A cancer microarray database and integrated data-mining platform. Neoplasia 2004:6:1-6.

21. Dalman MR, Deeter A, Nimishakavi G, Duan ZH. Fold change and p-value cutoffs significantly alter microarray interpretations. BMC Bioinformatics 2012;13 Suppl 2:S11.

22. McCarthy DJ, Smyth GK. Testing significance relative to a fold-change threshold is a TREAT. Bioinformatics 2009;25:765-71.

23. Christy HJ, Priyadharshini L. Differential expression analysis of JAK/ STAT pathway related genes in breast cancer. Meta Gene 2018;16:122-9.

24. Cerami E, Gao J, Dogrusoz U, Gross BE, Sumer SO, Aksoy BA, et al. The cBio cancer genomics portal: An open platform for exploring multidimensional cancer genomics data. Cancer Discov 2012;2:401-4.

25. Xia J, Gill EE, Hancock RE. NetworkAnalyst for statistical, visual and network-based meta-analysis of gene expression data. Nat Protoc 2015;10:823-44.

26. Aguirre-Gamboa R, Gomez-Rueda H, Martínez-Ledesma E, MartínezTorteya A, Chacolla-Huaringa R, Rodriguez-Barrientos A, et al. SurvExpress: An online biomarker validation tool and database for cancer gene expression data using survival analysis. PLoS One 2013;8:e74250.

27. Eswar N, Webb B, Marti-Renom MA, Madhusudhan MS, Eramian D, Shen MY, et al. Comparative protein structure modeling using MODELLER. Curr Protoc Protein Sci 2007;50:0209s50.

28. Eswar N, Webb B, Marti-Renom MA, Madhusudhan MS, Eramian D, Shen MY, et al. Comparative protein structure modeling using modeller. Curr Protoc Bioinformatics 2006; Chapter 5:Unit-5.6.

29. Kim S, Chen J, Cheng T, Gindulyte A, He J, He S, et al. PubChem 2019 update: Improved access to chemical data. Nucleic Acids Res 2019;47:D1102-D1109.

30. Daina A, Michielin O, Zoete V. SwissADME: A free web tool to evaluate pharmacokinetics, drug-likeness and medicinal chemistry friendliness of small molecules. Sci Rep 2017;7:42717.

31. Christy J. Computational analysis of disease associated nssnps in mmp2. Int J Pharm Bio Sci 2013;4:504-12

32. Christy HJ, Preethi B. Analyzing the effect of nssnps in cyp1a1 towards benzothiazoles binding. Int J Pharm Pharm Sci 2014;6:552-7.

33. Krammer A, Kirchhoff PD, Jiang X, Venkatachalam CM, Waldman M. LigScore: A novel scoring function for predicting binding affinities. J Mol Graph Model 2005;23:395-407.

34. Swift RV, Jusoh SA, Offutt TL, Li ES, Amaro RE. Knowledge-based methods to train and optimize virtual screening ensembles. J Chem Inf Model 2016;56:830-42

35. Epifano F, Curini M, Genovese S, Blaskovich M, Hamilton A, Sebti SM. Prenyloxy phenyl propanoids as novel lead compounds for the selective inhibition of geranylgeranyl transferase I. Bioorg Med Chem Lett 2007;17:2639-42.

36. Brooks B, Bruccoleri R, Olafson B, States DJ, Swaminathan S, Karplus M. CHARMM: A program for macromolecular energy, minimization, and dynamics calculations. J Comput Chem 1983;4:187-217.

37. Mortier J, Rakers C, Bermudez M, Murgueitio MS, Riniker S, Wolber G, et al. The impact of molecular dynamics on drug design: Applications for the characterization of ligand-macromolecule complexes. Drug Discov Today 2015;20:686-702. 